'Escuela de Medicina, Universidad Austral de Chile. Valdivia, Chile. 2Escuela de Medicina, Universidad de Concepción. Concepción,

Chile.

${ }^{3}$ Escuela de Antropología, Universidad Austral de Chile. Valdivia, Chile. anterna de Medicina. ${ }^{\mathrm{b}}$ Antropóloga.

Los autores declaran no poseer conflictos de interés. Los autores declaran no poseer fuentes de financiamiento externo.

Recibido el 2 de mayo de 2017, aceptado el 20 de octubre de

Correspondencia a: Diego E. Clouet-Huerta Ancha Sacha 371. Calama, Chile. dclouet@hotmail.com

\section{Especialización médica en Chile: tipos, mecanismos y requisitos de postulación. Una actualización de los procesos para los médicos generales}

\author{
DIEGO E. CLOUET-HUERTA ${ }^{1}$, BÁRBARA GONZÁLEZ ${ }^{2, a}$, \\ KATHERINE CORREA ${ }^{3, b}$
}

\section{Medical specialization in Chile. A centralized vision}

\begin{abstract}
Medical graduates face different postgraduate training options, but their priority is to obtain a primary medical specialty, defined as a specialty that does not derive from other. There are different specialty training programs in Chile, which can be dependent or independent of the Ministry of Health. The information about these programs is available in different Internet sites. However a centralized information service that groups and synthetize these programs is lacking, hampering graduate choice decisions. This article aims to review all specialization program modalities, providing a general vision of the institutional structure and implications that govern the specialization process in Chile.
\end{abstract}

(Rev Med Chile 2017; 145: 1454-1462)

Key words: Chile; Education, Medical; Specialization; Students, Medical.
E $\mathrm{n}$ las últimas décadas, Chile ha logrado un gran desarrollo económico y productivo, el que se ha traducido en mejoras sustanciales en variados aspectos, pero sobre todo en la salud de la población. El aumento en la esperanza de vida, la disminución en la mortalidad por enfermedades infectocontagiosas y las mejoras en el acceso a la salud, han incrementado la población del país y al mismo tiempo han generado una importante demanda para el sistema público de salud, sobrepasando su capacidad de atención ${ }^{1-3}$. Según un informe del Ministerio de Salud (MINSAL), en el año 2014 existía un déficit de 3.795 especialistas que afectaban a 1.500 .000 usuarios $^{4}$.

Con el fin de disminuir el déficit, se han desarrollado estrategias nacionales, como aumentar los cupos y centros formadores de médicos e incrementando el número de becas para especialidades médicas con devolución en el sistema público ${ }^{4}$.

Lo anterior ha generado múltiples opciones para la especialización médica primaria (EMP) en Chile, entendiéndose como la formación en especialidades médicas no derivadas de otras, por ejemplo, Medicina Interna o Pediatría, con procesos, requisitos y exigencias distintas para cada una. Algunos de estos aspectos son de conocimiento público pero otras desconocidos por los aspirantes, especialmente para los médicos recién egresados. Estos suelen recibir esta información de sus pares dadas las pocas fuentes de información formal y la escasa mención al respecto dentro de su formación académica, viendo reducidas sus opciones de EMP y desconociendo las implicancias de cada opción.

El presente trabajo tiene por objetivo revisar cada una de las modalidades por las cuales un médico general (MG) puede acceder a la EMP en nuestro país, a fin de proporcionar una visión general de la institucionalidad e implicancias que rigen el proceso en Chile. 


\section{Tipos de especialización médica}

En Chile, actualmente, existen seis modalidades por las cuales un MG puede acceder a una EMP, estas pueden clasificarse en dependientes e independientes del MINSAL (Figura 1).

\section{Dependientes del Ministerio de Salud}

Es importante aclarar que los programas de especialidades médicas son de responsabilidad exclusiva de los centros formadores quienes determinan el número de cupos de EMP y su distribución por modalidad, según su capacidad docente y las necesidades de la institución y del Estado, pero cumpliendo la normativa legal vigente del Comité Nacional de Acreditación.

Este tipo fue desarrollado a raíz del déficit de médicos generales y especialistas en el sistema público de salud y por la tendencia de estos a migrar al sistema privado por diversos motivos: carga asistencial, remuneraciones y prestaciones disponibles, entre otros.

En este contexto, desde 2015, el MINSAL ha desarrollado el plan de "Ingreso, Formación y Retención de Especialistas en el Sector Público de Salud", a través de una expansión presupuestaria progresiva y anual; buscando durante el período 2015-2018 formar 4.000 nuevos médicos especialistas para el sistema público ${ }^{5}$, mediante la incorporación de médicos a la atención primaria, la implementación de tecnología e infraestructura, mejorando las condiciones laborales y educando permanentemente en salud.

Dentro del ámbito formativo del plan señalado, el MINSAL asegura e incrementa progresivamente el número de cupos para EMP disponibles en las universidades formadoras. En el 2002 se

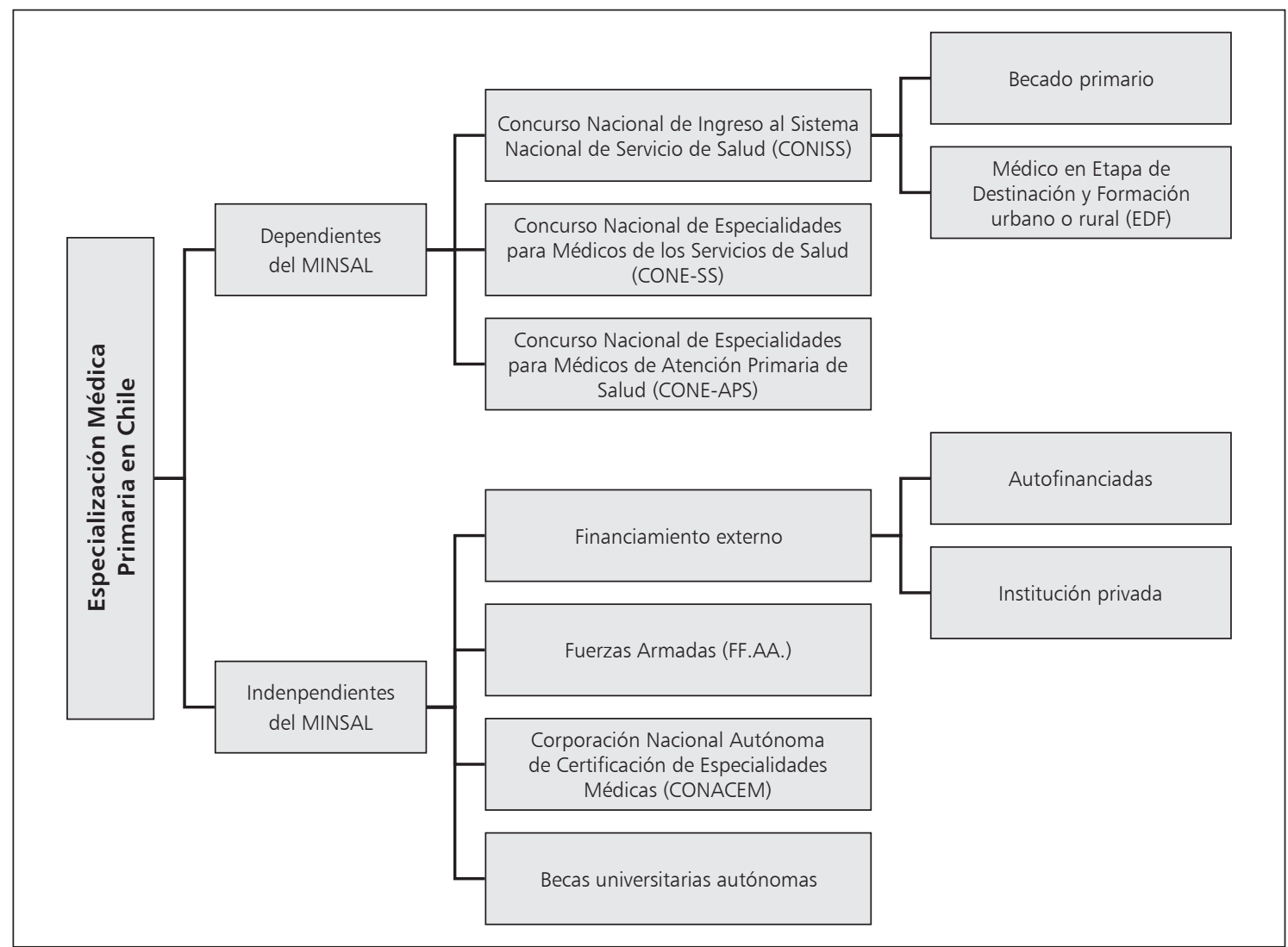

Figura 1. Mecanismos de ingreso a la especialización primaria en Chile el 2016. MINSAL: Ministerio de Salud de Chile; EDF: Dado mecanismo existente previamente es mejor conocido como Médico General de Zona (MGZ). 
formaron 150 médicos especialistas con recursos públicos, ascendiendo en 2015 a $1.128^{5}$, disminuyendo consecutivamente los cupos por otras vías de especialización, especialmente los autofinanciados, a fin de retener a los nuevos especialistas en el sistema público ${ }^{6}$.

Los cupos disponibles son distribuidos mediante tres procesos independientes, los cuales difieren en fechas de realización y tipos de aspirantes, sin embargo, poseen en común su respaldado por leyes nacionales, otorgando resguardos a los postulantes, pero también sanciones judiciales en caso de incumplimiento ${ }^{6}$.

\section{Concurso Nacional de Ingreso al Sistema Nacional de Servicios de Salud (CONISS)}

Está orientado a los médicos egresados en los dos últimos años en relación a la fecha del concur- so. Cuenta con la ventaja de disponer del mayor número de cupos para EMP y está regulado por la Ley No 19.964. Presenta dos vías para acceder a la EMP: Médico en etapa de destinación y formación (EDF) y becado primario ${ }^{7}$.

Dicha ley establece, al menos una vez por año, la realización de un proceso imparcial, público y nacional para acceder al CONISS. Considera una serie de requisitos mínimos de postulación y antecedentes académicos del candidato (Tabla 1 y Figura 2$)^{8}$ que son analizados y evaluados por una comisión de selección que asigna puntaje por diferentes ítems, pudiendo apelar ante fallos. Se elabora un listado definitivo en orden decreciente con los postulantes, según su puntaje y en una reunión de carácter presencial, cada uno es llamado para optar a los cupos en cada una de las vías. Existe una segunda convocatoria del con-

Tabla 1. Requisitos de postulación y antecedentes académicos solicitados en el Concurso Nacional de Ingreso al Sistema Nacional de Servicios de Salud (CONISS) de 2016

\begin{tabular}{|ll}
\hline $\begin{array}{l}\text { Requisitos de } \\
\text { postulación }\end{array}$ & - Nacionalidad chilena o certificado de residencia definitiva en Chile \\
& - diente \\
& - Acredificado original de egreso o título que incluya la Calificación Médica Nacional (CMN) \\
& - No haber optado a una beca de especialización, ni cargo en EDF a través del mismo proceso estable- \\
& cido en el artículo $8^{\circ}$ de la Ley 19.664 en algún proceso de selección anterior \\
Antecedentes & - Meses desempeñados como ayudante alumno \\
académicos & - Trabajos científicos \\
solicitados & - Practica de atención abierta \\
& - Cursos de capacitación y perfeccionamiento \\
& - Publicaciones científicas \\
& - Presentaciones en congresos
\end{tabular}

$$
\mathrm{CMN}=0,6 \times(\mathrm{NL})+0,3 \times(\mathrm{NI})+0,1 \times(\mathrm{NP})
$$

Nota de Licenciatura (NL): Corresponde al promedio aritmético de las asignaturas de primero a quinto año de Medicina, o bien, en caso del uso de créditos al promedio ponderado, este se calcula en base a la "nota de asignatura" multiplicado por el "número de créditos de la asignatura" y dividido por la "suma total de créditos de las asignaturas"

Nota de Internado (NI): Promedio aritmético de las notas de los cuatro internados básicos (Medicina, Obstetricia, Cirugía y Pediatría) más la de un quinto internado decidido por cada facultad (Internado rural, Psiquiatría, Especialidades, Optativo, etc.)

Nota de Pregrados (NP): Obtenido del promedio aritmético de los exámenes de pregrado de Medicina, Obstetricia, Cirugía y Pediatría

Figura 2. Fórmula para el cálculo de la Calificación Médica Nacional (CMN). Adaptado de Radrigan ME, Arteaga E. Calificación Médica Nacional. Rev Med Chile 1990; 118: 925-926. 
curso, de similar metodología, pero que dispone de menos cupos al ser los restantes de la primera convocatoria.

Los postulantes por vía becado primario, al momento de la convocatoria, deben estar necesariamente habilitados en el centro formador a postular, ya que paralelo al CONISS, cada centro posee una comisión evaluadora que determina la competencia del postulante a un programa de formación de especialista en la respectiva escuela de postgrado, mediante entrevistas personales, antecedentes académicos y pruebas psicológicas.

Dependiendo de la modalidad escogida, la iniciación de la formación varía. A continuación, se describen ambas.

\section{a. Médico en etapa de destinación y formación urbano o rural}

Popularmente conocido y denominado médico general de zona (MGZ), por el programa anterior de EMP, cuenta con dos etapas, una de destinación (ED) y otra de formación (EF).

La ED busca la incorporación de la última promoción de MG a centros de salud públicos situados en las localidades más vulnerables del país. Se ubican en sectores urbanos o rurales, poseen diferentes grados de complejidad y corresponden a: Centros de salud familiar (CESFAM), centros comunitarios de salud familiar (CESCOF) y hospitales de baja a moderada resolución; los centros, denominados "plazas", son categorizadas según variables sociodemográficas y epidemiológicas

\section{Tabla 2. Antecedentes solicitados del postulante a la etapa de formación del Médico en Etapa de Destinación y Formación (EDF) Urbano o Rural en Chile el 2016}

\footnotetext{
- Antigüedad en la EDF

- Nivel de dificultad de la plaza

- Funciones directivas

- Trabajo en posta rural

- Trabajo en servicios de urgencia

- Trabajos de investigación

- Cursos de perfeccionamiento

- Organización de actividades en la comunidad

- Actividades docentes

- Calidad de médico cirujano único
}

por letras desde la $\mathrm{A}$ a la $\mathrm{E}$ en orden decreciente de complejidad y puntaje.

Finalizada la ED, los médicos, mediante un concurso anual similar al realizado para ingresar al CONISS, postulan a las distintas EMP ofrecidas por el MINSAL. Considera un gran número de cupos, al ser el primer concurso del año calendario, dependiente del MINSAL en realizarse. Los postulantes deben entregar nuevos antecedentes (Tabla 2) que son evaluados por una comisión, que realiza un listado en orden decreciente de todos los candidatos según puntaje obtenido. Luego, en una convocatoria presencial, cada candidato selecciona una EMP según su lugar en el listado final. Se debe considerar que algunas universidades exigen que el postulante esté previamente habilitado en el centro. De esta forma, se da inicio a la EF.

Esta vía de EMP dura un período máximo de 9 años y, al respecto, es importante considerar tres puntos:

1. La permanencia mínima en la ED son 3 años $y$ a fines del tercer año se puede postular a la EF para el cuarto año.

2. El EDF dura como máximo 9 años, considerándose en ellos los años en ED y EF, implicando que, de postular al séptimo año a una especialidad de 3 años quedaría fuera del programa por exceder el período máximo.

3. Una vez cumplido los 9 años del EDF y, dado el término del contrato adquirido con el MINSAL se pierde el derecho a postular a una beca a través del programa.

Concluida la EF, y con ello el EDF, no existe obligatoriedad del ejercicio como especialista o "devolución" en el sistema de salud público.

Adicionalmente, esta vía presenta como beneficio la realización de carrera de funcionario, por considerar al profesional como trabajador público y mantener el sueldo recibido previamente, mientras se realiza la $\mathrm{EF}^{7}$.

\section{b. Becado primario}

Esta vía permite que el MG, ahora llamado becario, una vez adjudicado el cupo por el CONISS, ingrese directamente a su formación como especialista. Según lo dispuesto actualmente presenta dos períodos: El formativo (PF) y el asistencial obligatorio (PAO).

Durante el PF del becario, el MINSAL realiza el pago del arancel y matrícula, correspondientes 
al costo del programa fijado por la institución formadora, adicionalmente otorga un estipendio mensual, correspondiente al equivalente del sueldo base que recibe un profesional que cumple una jornada de $44 \mathrm{~h}$, siendo mayor en caso de especializaciones calificadas "de interés nacional" y, además, considera el derecho a recibir bonificaciones previsionales y asignación familiar en conformidad a la ley aplicable.

Finalizado el PF, e inmediatamente a continuación, el becario debe cumplir con el PAO, suscrito al momento de la adjudicación de la beca primaria. Este consiste en la obligación de desempeñarse en calidad de funcionario, como especialista, en un establecimiento del Sistema Nacional de Servicios de Salud por un lapso igual al doble de la duración de su PF. El establecimiento en el cual deberá desempeñarse queda definido al momento de la adjudicación del cupo en el "Convenio sobre derechos, obligaciones y garantía de becario en programa de especialización" celebrado entre el becario y la Subsecretaría de Redes Asistenciales, no permitiendo cambios posteriores del establecimiento, según lo definido por la Ley No 19.664. Este convenio también establece cláusulas penales y multas en caso de: Renuncias al programa de especialidad e incumplimiento del PAO, ya sea por inasistencia o abandono, inhabilitando, en este último caso, la contratación del médico dentro del servicio público de salud por un período de hasta 6 años $^{7}$.

\section{Concurso Nacional de Especialidades para} Médicos de los Servicios de Salud (CONE-SS)

Concurso para MG que se desempeñan en los servicios de salud públicos del país, facilitando la
EMP mediante un mecanismo similar al becado primario, consta de dos etapas: EF y ED.

Para postular a este proceso, los MG deben corresponder a la generación titulada a partir del tercer año previo al concurso y, según último proceso, hasta el 2006, variando esto según la versión del concurso; y que estén contratados por las leyes: No 19.378, Artículo 9o de la No 19.664 y No 15.076. En las primeras dos leyes se exige que los $M G$ se hayan desempeñado por un lapso no inferior a tres años en algún servicio de salud, o bien, de postular a especialidades médicas de interés nacional: Medicina Interna, Medicina Familiar, Obstetricia y Ginecología, Psiquiatría Adulto, Psiquiatría Pediátrica y de la Adolescencia, Pediatría, Salud Pública y Medicina de Urgencia, dicho período no podrá ser inferior a un año. Para Medicina de Urgencia, esta exige como requisito adicional una edad inferior a 35 años y haber ejercido como MG por más de 4 y menos de 5 años desde la fecha de titulación hasta el año previo del concurso.

Para la selección de postulantes se evalúan sus antecedentes, considerando dos ejes: Desempeño Asistencial o Laboral y Desempeño Académico (Tabla 3). Se realiza un listado final en orden decreciente de acuerdo al puntaje obtenido, convocándose una reunión presencial para la adjudicación de los cupos. Los centros formadores establecen requisitos de ingreso o permanencia, solicitando la habilitación en el centro previa adjudicación del cupo.

Luego de la EF, inicia la ED, que corresponde a la "devolución" en un centro establecido durante la adjudicación del cupo, por un tiempo equivalente al doble de la duración de la EF, siendo

\section{Tabla 3. Rubros evaluados en el Concurso Nacional de Especialidades para Médicos de los Servicios de} Salud (CONE-SS). Chile, 2016

\begin{tabular}{|ll|}
\hline $\begin{array}{l}\text { Desempeño } \\
\text { asistencial o } \\
\text { laboral }\end{array}$ & - Desempeño como médico en Atención Primaria de Salud \\
& - Desempeño en establecimientos de uno o más servicios de salud por Ley No 15.076 \\
& - Desempeño como médico en COMPIN, SEREMI o SML \\
Desempeño & - Desempeño en instituciones docentes \\
académico & Cursos de capacitación y perfeccionamiento y postgrado \\
& - Examen Único Nacional de Conocimientos de Medicina (EUNACOM) o examen médico nacional (EMN) \\
\hline
\end{tabular}

UCI: Unidad de Cuidados Intensivos; UTI: Unidad de Tratamiento Intermedio; COMPIN: Comisión de Medicina Preventiva e Invalidez; SEREMI: Secretaria Regional Ministerial; SML: Servicio Médico Legal. 
posible validar hasta el 50\% del tiempo de la ED, según los años trabajados en el servicio público al momento de la postulación al CONE-SS ${ }^{9}$.

\section{Concurso Médicos Atención Primaria de Salud (CONE-APS)}

Proceso destinado a MG que se desempeñan en atención primaria de salud, contratados bajo la ley No 19.378 o por el artículo 9 de la Ley No 19.664 , siendo esta última la que identifica a los médicos contratados por los directores de los servicios de salud como profesionales en EDF. Este proceso consta de dos etapas: EF y PAO.

Entre los requisitos de postulación se incluye el tiempo de desempeño previo, dependiendo de la especialidad a postular, siendo requisito un lapso no inferior a un año a la fecha de postulación en el nivel primario de uno o más servicios de salud o establecimientos de salud municipal para los aspirantes a especialidades de "interés nacional", excluyendo medicina de urgencia y medicina familiar; otras especialidades requieren de un período mínimo de 3 años a la fecha de postulación. Entre los rubros evaluados al postular (Tabla 4), los ítems de mayor puntuación son: antigüedad funcionaria, trabajo comunitario, cursos de perfeccionamiento, trabajos de investigación y docencia.

Al igual que en los concursos previos, una vez elaborada la lista de puntajes definitivos, se procede al ofrecimiento de los cupos de especia-

\section{Tabla 4. Rubros evaluados en el Concurso Médicos Atención Primaria de Salud (CONE-APS). Chile, 2016}

\section{- Antigüedad funcionario \\ - Cursos de perfeccionamiento \\ - Funciones de responsabilidad \\ - Organización de proyectos y trabajo con la comunidad en centros comunitarios o postas rurales \\ - Trabajos de investigación o docencia \\ - Servicios de urgencia o extensión horaria \\ - Calificación médica nacional \\ - Desempeño en zonas extremas}

Zonas Extremas: Regiones de Arica y Parinacota, Tarapacá, Aysén, Magallanes y la Antártica Chilena; y Provincias de Chiloé y Palena. lización mediante un llamado a viva voz, el cual se realiza en las mismas dos instancias. Es necesario previamente la habilitación del postulante en el centro formador.

El proceso tiene una duración máxima de 9 años en total, correspondiendo a la sumatoria de los períodos en EF y PAO. Se debe destacar que al momento de adjudicación del cupo del concurso es posible validar hasta el $50 \%$ del tiempo desempeñado previamente. Durante la EF se mantiene la calidad funcionaria y remuneración conservando su contrato durante el ciclo ${ }^{10}$.

\section{Especialización médica independiente del Ministerio de Salud}

En general, son las vías para la EMP menos conocidas, corresponden a procesos regulados por contratos entre sus participantes. Cada una presenta fechas y requisitos de postulación diferentes, siendo aconsejable la consulta periódica de la información.

\section{Financiamiento externo}

Existen dos opciones de financiamiento externo disponibles para los médicos generales que pudiesen financiar el costo del programa, ya sea de forma independiente o respaldados por instituciones privadas, clínicas o mutuales de seguridad, correspondiendo a las autónomas o con financiamiento privado, respectivamente. La primera tiene la ventaja de que el becario, una vez completado el período de formación, no debe realizar compensación alguna al sistema público o privado y su desventaja es el no recibir un estipendio de manutención durante su formación; la segunda, por el contrario, al depender de un centro privado, una vez completada la formación debe realizar una devolución a la institución, y el tiempo adeudado u otras cláusulas adicionales dependerán del acuerdo acordado entre las partes variando evidentemente en cada caso.

Últimamente, el número de postulantes a esta modalidad ha ido decreciendo. Este fenómeno se debe, en parte, a la disminución de cupos y centros de formación con esta opción, como también por el elevado costo de los programas, fijado por cada institución. A modo de ejemplo, y dado variabilidad del costo anual, para el año 2017, el costo anual de formar un traumatólogo en la Univer- 
sidad Austral de Chile corresponde a \$ 5.800.000 pesos chilenos, mientras que el de un médico internista en la Universidad de Valparaíso es de aproximadamente $\$ 5.542 .320$ pesos chilenos ${ }^{11,12}$. Cada año, usualmente en diciembre, las instituciones publican en sus páginas electrónicas los cupos disponibles, requisitos y costos asociados.

\section{Fuerzas Armadas}

En Chile existe la posibilidad de ingresar como MG en grado de Oficial de Sanidad a las Fuerzas Armadas (FF.AA.), en las distintas ramas: Ejército, Armada o Fuerza Aérea; desempeñándose en centros de salud al interior de la institución, zonas de conflicto o misiones de paz. Luego de un período mínimo de 5 años dentro de la institución, se puede postular a una EMP recibiendo el financiamiento de $100 \%$ del programa de formación por parte de la institución. Posterior a ello, exige la devolución mínima por 5 años como especialista en las FF.AA, con la posibilidad de trabajar paralelamente fuera de la institución. Una vez finalizada la devolución se puede continuar trabajando o solicitar la baja de la institución.

Cada una de las ramas de las FF.AA publica, usualmente en diciembre, en sus páginas electrónicas la información referente a los requisitos $y$ antecedentes necesarios (Tabla 5). El proceso de selección incluye una entrevista personal, un examen médico que acredite salud compatible del postulante y un examen práctico de conocimientos en medicina.

Respecto a las especialidades disponibles para postular, si bien son cupos ilimitados, varían anualmente según las necesidades de la institución; pese a esto, se puede solicitar la evaluación de otras especialidades. Destaca el beneficio de la mantención del sueldo como oficial durante la formación, desarrollo de carrera militar y disponibilidad para realizar cursos como: medicina subacuática e hiperbárica, medicina aeroespacial, entre otros.

\section{Corporación Nacional Autónoma de Certificación de Especialidades Médicas}

Este mecanismo permite que los MG que se encuentren desempeñándose en distintos servicios de especialidades del país puedan, tras cumplir un período de adiestramiento en práctica (AP), ser reconocidos como especialistas, siendo la Corporación Nacional Autónoma de Certificación de Especialidades Médicas (CONACEM) la corporación de derecho privado encargada de esto ${ }^{14}$.

Para iniciar este proceso se debe recopilar y enviar antecedentes a la corporación, además de pagar un costo que asciende a $\$ 180.000$ o \$ 200.000 pesos chilenos, según sea la certificación en un centro nacional o extranjero, respectivamente.

Existen requisitos generales para la postulación (Tabla 6), y otros específicos según la especialidad a certificar, los que se detallan en su página elec-

Tabla 5. Requisitos y antecedentes solicitados para el ingreso como Oficial de Sanidad en las Fuerzas Armadas (FF.AA.) de Chile, 2016

\begin{tabular}{|ll|}
\hline Requisitos & - Nacionalidad chilena \\
- & Presentar menos de 35 años de edad \\
- & Título de médico cirujano \\
- & Disponibilidad inmediata de traslado a cualquier área del país o extranjero \\
- & Curriculum vitae \\
- Puntecedentes & - Concentración notas universidad \\
- Certificado título profesional \\
- Nota de titulación carrera \\
- Nota del examen único nacional de conocimientos de medicina (EUNACOM) \\
- Cursos efectuados \\
- Trabajos de investigación publicados \\
- Post grados efectuados \\
- Carta motivacional \\
\hline
\end{tabular}


Tabla 6. Requisitos generales solicitados para la certificación de especialidades a través de la Corporación Nacional Autónoma de Certificación de Especialidades Médicas (CONACEM), 2017

1. Título de Médico Cirujano:

Obtenido en universidades chilenas

Legalmente validado de corresponder a universidades extranjeras

2. Acreditar un período de adiestramiento en práctica:

- Período ininterrumpido de 5 años y 22 h semanales mínimo

- Realizado en hospital de alta complejidad perteneciente al ministerio de salud, o bien, un centro docente asistencial de igual complejidad que quedará a evaluación por el comité

- El centro debe poseer al menos dos especialistas, certificados por programas universitarios acreditados o por CONACEM, que avalen la formación y desarrollo del postulante

3. Cumplir con condiciones especificas de la especialidad a acreditar, disponible en el sitio web de CONACEM (http://www.conacem.cl/requisitos_especialidad.asp)

4. Aprobar las pruebas teóricas y prácticas diseñadas por CONACEM para validar formación como especialista

trónica ${ }^{15}$. Una vez certificada la especialidad no se debe realizar un período de devolución como la PAO.

\section{Becas Universitarias Autónomas}

Esta modalidad permite que los distintos centros formadores dispongan de cupos de EMP, de acuerdo a las necesidades del plantel, hacia los médicos cirujanos. Los requisitos de ingreso y devolución de esta modalidad son propios de cada centro, siendo modificados anualmente e informados a los aspirantes usualmente a finales de cada año a través de sus páginas electrónicas.

En líneas generales, esta modalidad ofrece a los becarios aceptados el financiamiento total del programa de especialización y, en algunos casos, un estipendio mensual asociado a modo de mantenimiento durante el período formativo, pero a cambio que posteriormente el becado trabaje al interior de la institución como docente o especialista.

Cabe destacar el caso particular de la Universidad de Chile con su opción de "Doctorado en Ciencias Médicas y Especialidad" que permite, por un lado adquirir los conocimientos y técnicas de un área específico para desarrollar su práctica asistencial y por otro la obtención de un título académico que le permita el desarrollo de las ciencias en un plazo de seis años donde ambas opciones se cursan en paralelo.

\section{Conclusión}

La EMP ha adquirido una categoría de interés nacional, transformándose en el eje central de desarrollo, un método para mejorar y disminuir las brechas sanitarias existentes en Chile. En comparación a décadas previas, han aumentado enormemente los mecanismos de especialización y anualmente se crean o modifican los ya existentes, generando diversos caminos con características totalmente distintas entre ellas, aumentando las posibilidades para los médicos.

Por lo anterior, y como repuesta al gran dinamismo del tema, esta revisión busca contribuir a una mejor comprensión del sistema actual de especialización, centralizando la información y haciéndola más amena tanto para los médicos recién egresados como para aquellos en formación, además de fomentar la discusión para mejorar su desarrollo y así alcanzar la meta de un sistema salud público de excelencia.

\section{Referencias}

1. Medina E, Kaempffer R. Tendencias y característivas de la mortalidad chilena 1970-2003. Rev Med Chile 2007; 135(2): 240-50.

2. Instituto Nacional de Estadísticas de Chile. Evolución de la Mortalidad en Chile según causas de muerte y edad, 
1990-2007. Santiago de Chile: Instituto Nacional de Estadísticas de Chile; 2010 [Acceso el 12 de diciembre de 2017]. Disponible en: http://historico.ine.cl/canales/ menu/publicaciones/calendario_de_publicaciones/ pdf/010211/evo90_07_010211.pdf

3. Barber R, Fullman N, Sorensen R, Bollyky T, McKee M, Noite E et. al. Healthcare Access and Quality Index based on mortality from causes amenable to personal health care in 195 countries and territories, 1990-2015: a novel analysis from the Global Burden of Disease Study 2015. Lancet 2017; 10091 (390): 231-66.

4. Ministerio de Salud de Chile. Informe sobre brechas de personal de salud por Servicio de Salud. Santiago de Chile: Ministerio de Salud de Chile; 2016 [Acceso el 23 de abril de 2017]. Disponible en: http://web. minsal.cl/wp-content/uploads/2015/08/Informe-Brechas-RHS-en-Sector-P\%C3\%BAblico_Marzo2016.pdf

5. Plan de ingreso, formación y retención de especialistas en el sector público de salud [Internet]. Santiago de Chile: Ministerio de Salud de Chile; 4 de agosto de 2015 [Acceso el 23 de abril de 2017]. Disponible en: http:// www.minsal.cl/medicosespecialistas/

6. Concursos 2016-2017 [Internet]. Santiago de Chile: Ministerio de Salud de Chile; 22 de septiembre de 2016 [Acceso el 23 de abril de 2017]. Disponible en: http:// web.minsal.cl/concursos-2016-2017/

7. Concurso Nacional de Ingreso al Sistema Nacional de Servicios de Salud (CONISS) [Internet]. Santiago de Chile: Ministerio de Salud de Chile; 20 de septiembre de 2016 [Acceso el 29 de abril de 2016]. Disponible en: http://web.minsal.cl/concurso-nacional-de-ingreso-al-sistema-nacional-de-servicios-de-salud-coniss/

8. Radrigan ME, Arteaga E. Calificación Médica Nacional. Rev Med Chile 1990; 118: 925-6.

9. Concurso Nacional de Especialidades para Médicos de los Servicios de Salud (CONE-SS) [Internet]. Santiago de Chile: Ministerio de Salud de Chile; 20 de septiembre de 2016 [Acceso el 29 de abril de 2016]. Disponible en: http://web.minsal.cl/concurso-nacional-de-especialidades-para-medicos-de-los-servicios-de-salud-cone-ss/

10. Concurso Médicos Atención Primaria de Salud año 2017 (CONE-APS) [Internet]. Santiago de Chile: Ministerio de Salud de Chile; 20 de septiembre de 2016 [Acceso el 29 de abril de 2016]. Disponible en: http://web. minsal.cl/concurso-medicos-atencion-primaria-de-salud-ano-2017/

11. Especialidades médicas [Internet]. Valdivia: Universidad Austral de Chile; 14 de diciembre de 2016 [Acceso el 5 de enero de 2017]. Disponible en: http://medicina.uach. cl/noticia.php?id=2641

12. Especialidad en Medicina Interna [Internet]. Valparaíso: Universidad de Valparaíso; [Acceso el 23 de abril de 2017]. Disponible en: http://www.uv.cl/postgra$\mathrm{do} / ? \mathrm{id}=167$

13. Admisión Médicos Navales 2017 [Internet]. Valparaíso: Armada de Chile; [Acceso el 23 de abril de 2017]. Disponible en: https://www.admisionarmada.cl/admision-medicos-navales-2017/admision/2016-10-12/095901.html

14. Corporación Nacional Autónoma de Certificación Especialidad Médicas (CONACEM) [Internet]. Santiago: CONACEM [Acceso el 25 de agosto de 2017]. Disponible en: http://www.conacem.cl/index.asp

15. Requisitos por especialidad para la certificación de especialistas [Internet]. Santiago: CONACEM [Acceso el 25 de agosto de 2017]. Disponible en: http://www.conacem. cl/requisitos_especialidad.asp

16. Doctorado en Ciencias Médicas y Especialidad [Internet]. Santiago de Chile: Universidad de Chile; 12 de diciembre de 2016 [Acceso el 23 de abril de 2017]. Disponible en: http://www.uchile.cl/postgrados/10286/ ciencias-medicas-y-especialidad 